\title{
ON THE POSSIBLE MECHANISMS OF THE FORMATION OF PIEZOELECTRIC ACTIVE ROCKS WITH CRYSTALLOGRAPHIC TEXTURES
}

\author{
A. N. NIKITIN and T. I. IVANKINA* \\ Joint Institute for Nuclear Research, Dubna, Russia \\ *Teacher's Training University, Tula, Russia
}

(Received 20 June 1995)

\begin{abstract}
The volume piezoelectric effect in polycrystalline rocks occurs if, in addition to preferred orientations of electric axes of crystals, these axes have orientational polarity. The present paper allows conclusions about some peculiarities of the processes and mechanisms leading to the formation of texturized rocks with piezoelectric properties. Information on the textures of rocks with peizoelectric activity was obtained by means of neutron diffraction, which gives the most complete information on the textures of large polycrystalline samples. These data, in combination with sample electrometric measurements, make possible the explanation of, for example, how the electric axes of quartz grains aligned in polycrystals. Three practicable mechanisms are suggested.
\end{abstract}

KEY WORDS: Piezoelectric effect, quartz, rocks, texture formation, neutron diffraction, pole figures, electrometric measurement.

\section{INTRODUCTION}

In 1954, M. P. Volarovich and E. I. Parkhomenko discovered the piezoelectric effect in rocks (Volarovich et al., 1954). The piezoelectric effect in polycrystals (in particular, in rocks) was explained on the basis of a theory proposed by A. V. Shubnikov (1946).

Usually, polycrystalline rocks, which are composed of piezoelectric active minerals, do not display the piezoelectric effect. For example, monomict sandstones are mainly composed of randomly oriented quartz grains, and as a result, the volume piezoelectric effect of a sample is zero.

Thus, to obtain a non-zero piezoelectric effect, the rock constituents should have lattice preferred orientation. However, monomineralic vein quartzites from many deposits have pronounced sharp textures, but none of them shows piezoelectric activity. The volume piezoelectric effect in polycrystalline rocks is manifested if, in addition to preferred orientations of electric axes of crystals, the orientational polarity of electric axes is observed.

The piezoelectric effect of rocks is widely used in geophysics. Unfortunately, no theory or statistical model on the formation of piezoelectric active texturized rocks exists yet. This is due to the lack of experimental information and due to restricted knowledge, how the piezoelectric properties of rocks are related to the conditions and mechanisms of rock genesis. 


\section{EXPERIMENTAL DETERMINATION OF THE TEXTURE-INDUCED PIEZOELECTRIC EFFECT}

Several investigators concluded, that it is impossible to measure the volume piezoelectric effect of rocks on the basis of measurements of the electric polarization of rock samples by static methods (Tyck et al., 1977). It is necessary to have methods for unambigous determination of the texture-induced piezoelectric effect, as well as the uncompensated effect caused by single grains.

To uncover the origin of the piezoelectric effect in inhomogeneous polycrystalline aggregates several unique properties of piezoelectric crystals are used:

- If elastic deformation changes from compression to extension, then the polarization charge sign also changes. This case is called static. The analogous situation is observed in the case of dynamical stimulation of the piezoelectric effect.

- Another evidence of the piezoelectric origin of the effect in rocks is the establishment of the so-called transverse piezoelectric effect, which may be observed, when the rock sample is switched on as an acoustic radiator, i.e., acoustic oscillations of the sample are registrated at irradiating the sample with electric signals.

- In addition we worked out a method to determine of the piezoelectric effect in polycrystals caused by textures. It is based on the regular distribution of the polarization charge density over the sample surface under static loading (or dynamic exciting). The comparison of the measured angular dependences of the piezoelectric effect with theoretical cross-sections of indicative surfaces allows conclusions on the texture-induced piezoelectric effect and also permits the determinations of the symmetry type of the piezoelectric properties (Nikitin et al., 1981; Nikitin, Parkhomenko, 1982b).

The piezoelecric effect caused by single grains in polycrystals without textures has distinguishable features and can be easily measured (Nikitin, Parkhomenko, 1982a). The complete information on textures of rocks can be obtained by using neutron diffraction method (Wenk, 1993).

\section{EXPERIMENTAL DATA ON PIEZOELECTRIC ACTIVE ROCKS}

A large set of quartz rock samples were chosen to investigate the piezoelectric active rocks. The experiments were carried out at the NSHR neutron diffractometer at the pulsed reactor IBR-2 of the JINR, Dubna (Walther et al., 1993). The piezoelectric effect was measured by the electrometric method using a point movable electrode following the acoustic excitation of the sample (Nikitin et al., 1981).

A vein quartz sample from a gold-bearing deposit demonstrated high piezoelectric activity. Figure 1 represents eight pole figures obtained by time-of-flight neutron diffraction (Walther et al., 1990; Ivankina et. al., 1991). The pole density distributions are characterized by sharp preferred orientations, the maxima of the $\{10 \overline{1} 1\}$ and $\{11 \overline{2} 0\}$ pole figures are located at an angular distance of $60^{\circ}$ from each other. They correspond to the electric quartz axes. Such density distributions are usually interpreted as an indication to the presence of a symmetry axis of the $6^{\text {th }}$ or $3^{\text {rd }}$ order. 

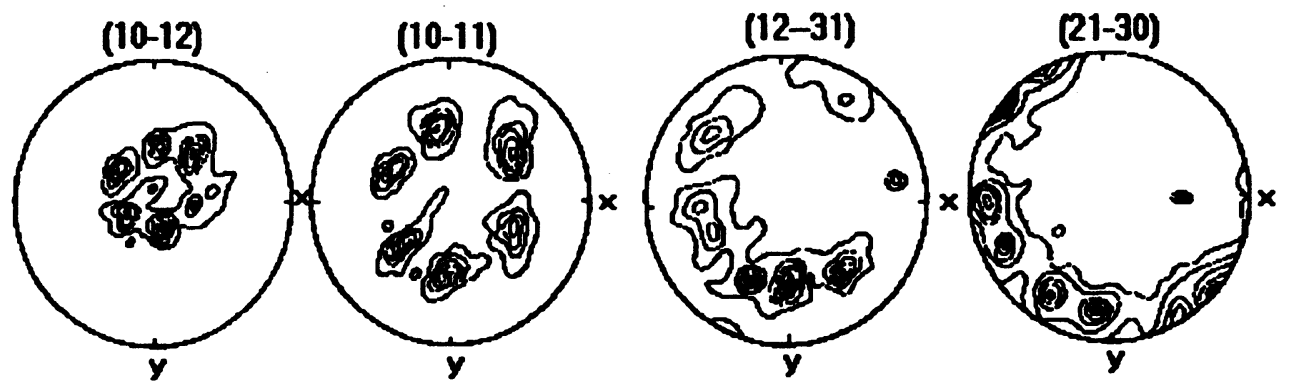

(11-20)

(11-22)

(20-21)

(11-21)
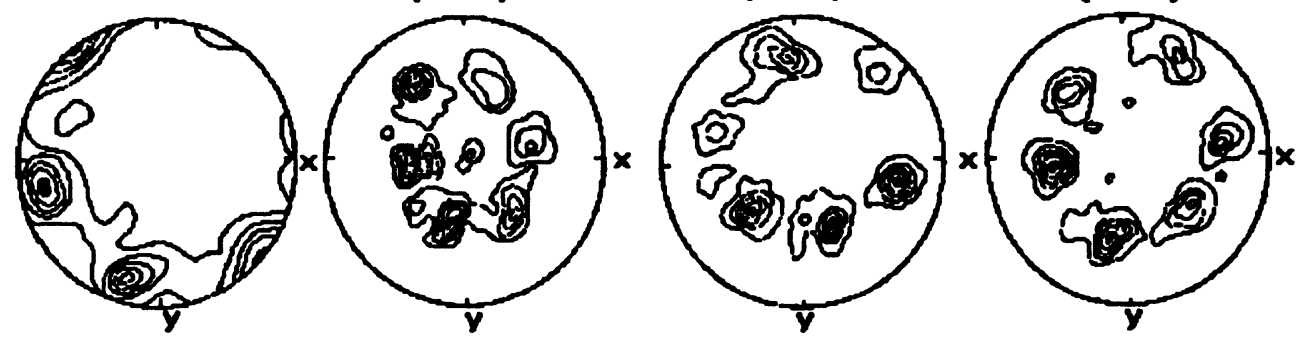

Figure 1 Pole figures of vein quartz measured by neutron diffraction.

In addition, the angular dependences of the piezoelectric field were registered (see Figure 2). Comprising the results of the texture and electrometric measurements we observe, that the symmetry of the texture correspond to the symmetry of the sample's electric field. The sample was piezoelectrically active and its symmetry axis is normal to the XY-plane. Rotation about an angle of $120^{\circ}$ around an axis normal to the XYplane is considered to represent physically invariant transformation. This fact proves that the symmetry axis is 3-fold.

The presented complex of experimental data permits to characterize the piezoelectric activity of a rock sample as a result of lattice preferred orientations.

The statistic analysis of the electrometer and neutron measurements from a large series of samples $(>100)$ revealed general texture features of the quartz-bearing rocks. The pole figures measured by neutron diffraction are used as a basis for the construction of models of texturized quartz aggregates.

These models take into account the fact that the properties and fabrics of ideal texturized aggregates depend on the properties and symmetry of constituent crystals. The models are developed for the statistic equilibrium of two enantiomorphic quartz modifications. A block of two single crystals of different modifications is selected as unit structural cell of a polycrystalline aggregate. It has been demonstrated that four different combinations of the unit cells construct four types of texturized aggregates corresponding to rocks with the symmetry of piezoelectric properties $(4 \cdot \mathrm{m}),(\mathrm{m})$, $(\mathrm{m} \cdot 3: \mathrm{m})$ and $(2 \cdot \mathrm{m})$ (in accordance to the Shubnikov terminology). Figure 3 shows the $\{0001\}$ and $\{11 \overline{2} 0\}$ simulated pole figures and crystallographic models for four texture types. It can be said with certainty that textures of the four presented types are most widespread in piezoelectric active vein quarts. 


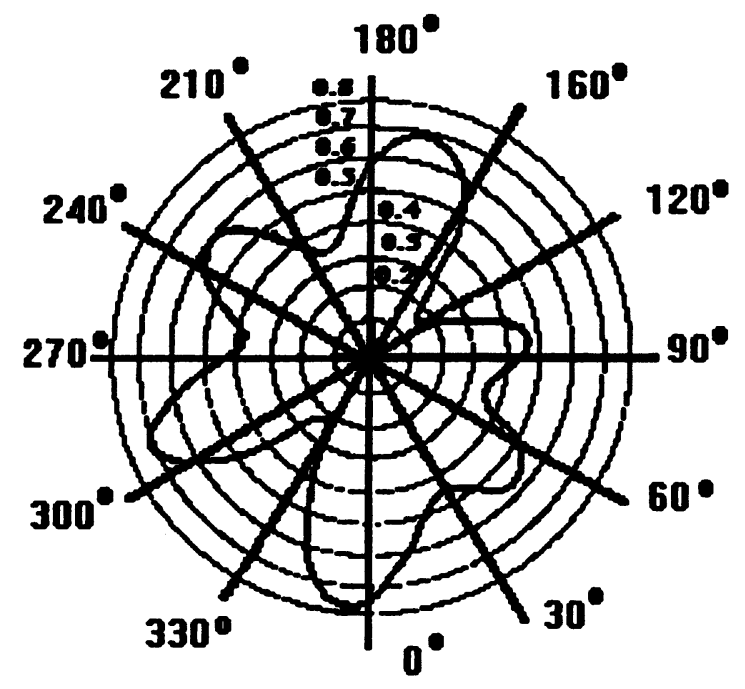

Figure 2 Angular dependences of electric field potentials $(\mathrm{mV})$ recorded in a vein quartz. Cylinder axes are normal to the electrical axes.

\section{ON THE GENETIC CLASSIFICATION OF ROCK TEXTURES}

It is well known that textures may develop as a result of crystallization, sedimentation, plastic deformation, recrystallization and metamorphism. If the main texture forming mechanisms are taken as a basis for texture classification, the following textures types can be outlined:

- growth (or crystallization) texture

- deformation texture

- recrystallization texture

- phase transformation texture

- sedimentation texture

The texture of rocks with piezoelectric properties can't be counted to any above mentioned class.

Each of the above-mentioned physical mechanisms of texture formation is controlled by thermodynamic parameters and external physical fields. The formation of growth textures during crystallization and of recrystallization textures depends on temperature variation and temperature gradients. In addition, pressure acting on materials significantly influences the texture types formed during phase transformation. It seems most likely that the stress-strain state of the material under external mechanical action influences the formation process and the resulting type of texture.

When textures of geomaterials are formed, the external shape of material changes, and this change is accompanied by a change in the orientation of the crystallographic lattices of minerals, which results in specific textures in accordance with the symmetry of the stress tensor. The orientations of the recrystallized grains depend on the loading 

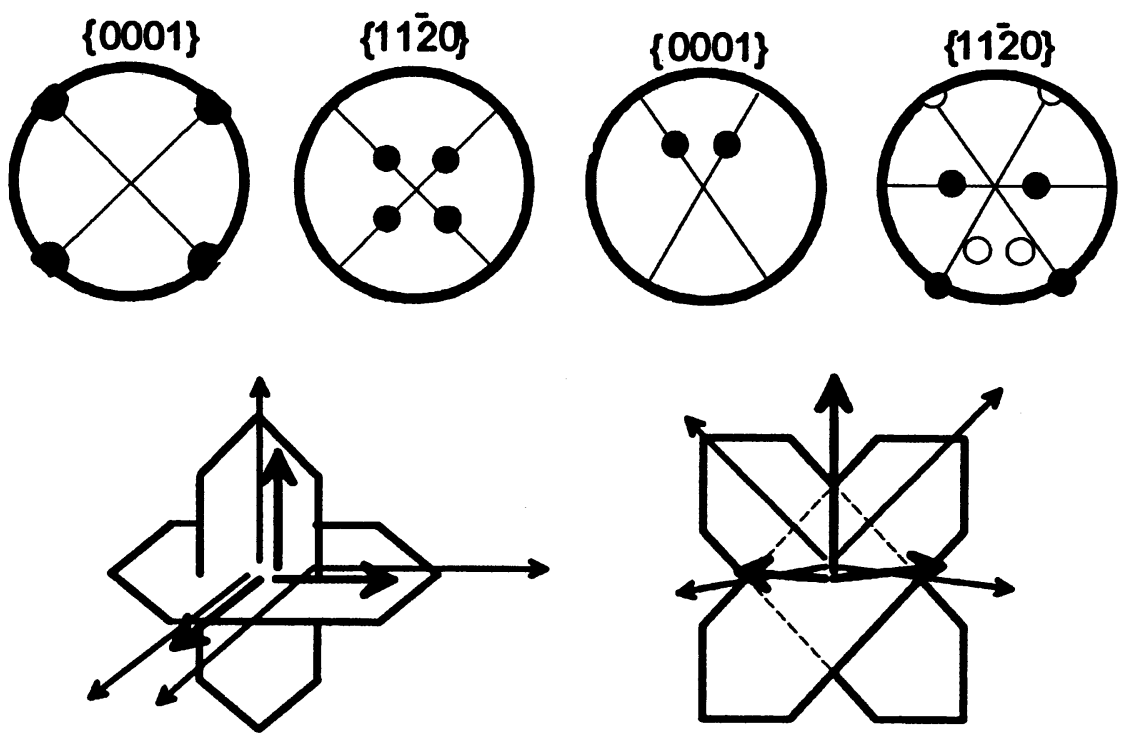

a
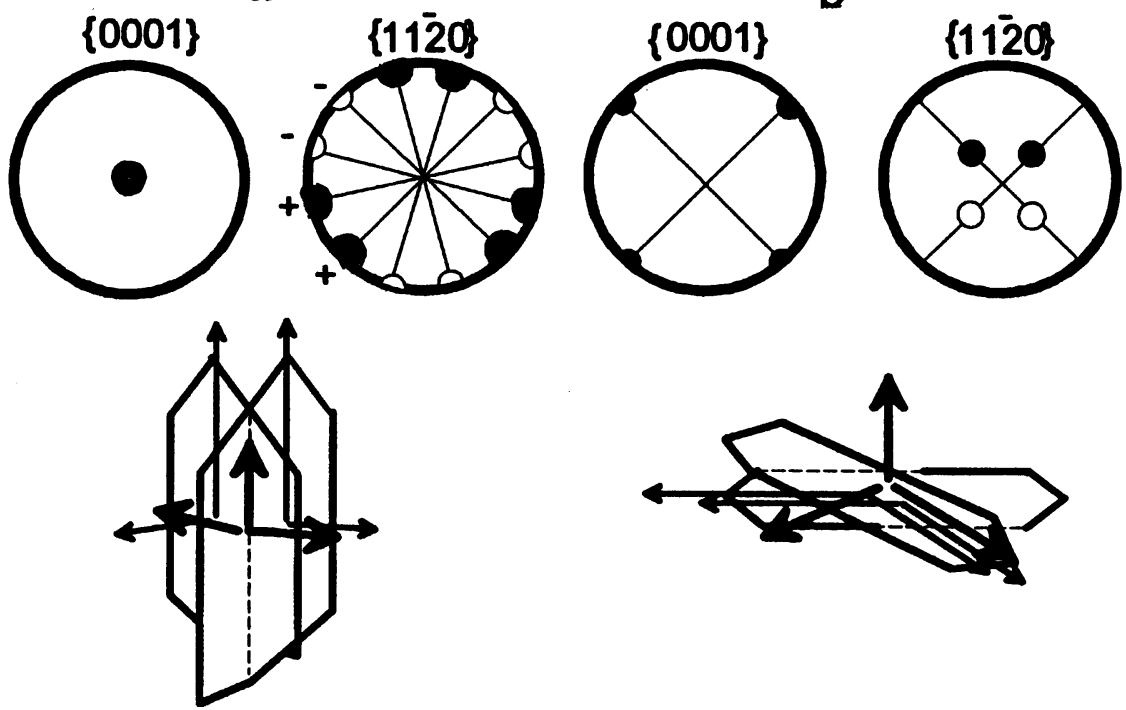

C

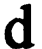

Figure $3\{0001\}$ and $\{11 \overline{2} 0\}$ pole figures and crystallographic models of ideal texturized quartz aggregates corresponding to rocks with the following symmetry types of piezoelectric properties (a) (4 - m); (b) (m); (c) (m·3:m); (d) (2 - m). Full circles in the $\{0001\}$ pole figures denote the orientation of the maxima of the optical axes. In the $\{11 \overline{2} 0\}$ pole figures full and open circles denote the orientations of positive and negative directions of the electrical axes. 
type in the static and dynamic recrystallizations. The orientation effect of mechanical stresses on the polytwin structures generated following the martensite phase transformation is illustrated on the basis of thermodynamic analysis (Royburt, 1983).

Bishop (1981) was the first to suggest a mechanism of piezoelectricity origination in rocks.

\section{POSSIBLE MECHANISMS OF THE FORMATION OF PIEZOELECTRIC ACTIVE ROCKS}

\section{Piezoelectricity of rocks with growth textures}

In polycrystalline quartz rocks preferred orientations develop as a result of geometric and crystallographic selection during crystallization. The druse quartz type with preferred orientations of the $\{0001\}$ crystallographic planes occurs very often. A preferred orientation of electric axes during the growth process is probable, but formation of causes for the polarity of the parallel axes of neighbouring grains must be clarified.

Let us consider the most probable situation of the growth of a large number of crystals from a solution. Figure 4 gives the growth surface cross sections of quartz, which is cut parallel to the $\{0001\}$ plane. As may be seen, the growth rates of single quartz crystals along polar axes in the $+x_{1}(+\{\overline{2} 110\})$ and $-x_{1}(-\{\overline{2} 110\})$ directions differ by approximately 3 times (Zinober et al., 1972). Therefore, if nucleus grain orientations and the character of their interaction cause preferred growth of an ensemble of crystallites along polar directions, then the volume fraction of grains growing in the positive direction will predominate over that in the negative direction (see Figure 5). This situation is observed in the case of statistical equality of grains, whose positive and negative directions align with the growth direction. If the number of nucleus grains with the positive direction of electric axes oriented parallel to the growth direction becomes larger, then the "grown" texture initiates an increase of piezoelectric activity.

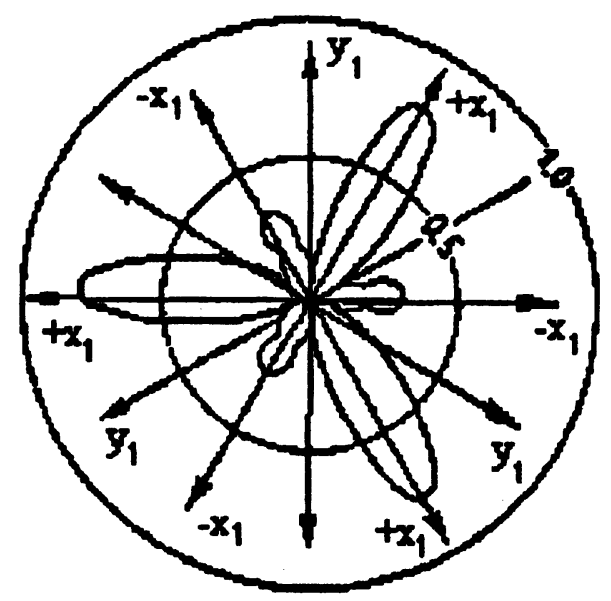

Figure $4\{0001\}$ cross-section of the quartz growth rate surface (mm/Day). 


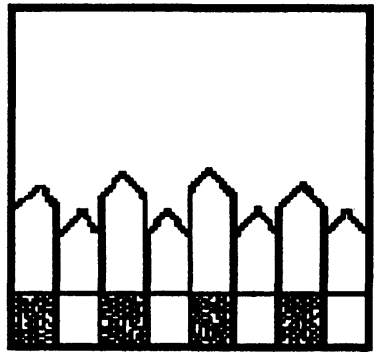

a

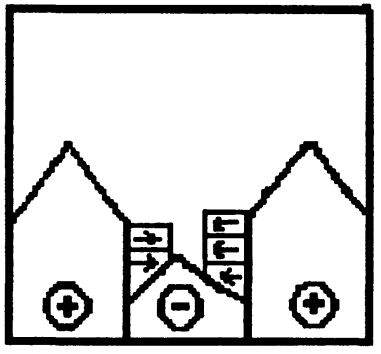

b

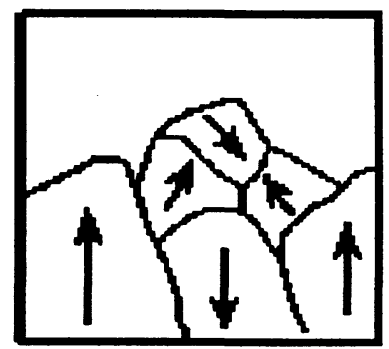

C

Figure 5 Development of piezoelectric active aggregates with growth texture (a,b,c) from a hydrothermal solution.

The feasibility of an experiment to test the probability of a rise of a similar "piezoelectric" texture from a hydrothermal solution is to be demonstrated. From coarsegrained vein quartz with a pronounced texture a cylinder sample of $30 \mathrm{~mm}$ in diameter and $5 \mathrm{~mm}$ height was cut. The base surface of the cylinder was oriented in such a way, that the principal electric axis of the majority of oriented crystals forms a small angle to the cylinder radius or is aligned with it. Then, the cylinder was placed into an autoclave, and a constant supersaturation gradient was maintained in the autoclave by fixing the temperature gradient between crystallization zones and solution zones of the initial mixture. The sample before the experiment is shown in Figure 6a. The process in the autoclave went on for over 7 weeks.

As a result, over the entire surface of the smooth sample the new crystalline "forest" has grown (figure 6b). The newly grown druse is characterized, first, by the fact that the largest part of the new crystals have clearly preferred orientations, second, some groups of crystals have distinguishably different sizes which might be due to different growth rates along the positive and negative directions of the quartz electric axes.

\section{Piezoelectricity of rocks with deformation textures}

Let us assume, that a massif of quartz rocks or vein quartz was formed under high (above the $\beta-\alpha$ phase transition) temperature and underwent plastic deformation. The development of preferred orientations in such rocks is commonly observed. Probable types of $\beta$-quartz textures formed during pure shear and uniaxial compression were simulated using the thermoactive variant of the Taylor theory (Walther et al., 1994, Ivankina, in press).

If the temperature decreases below $573^{\circ}$ in such piezoelectric neutral texturized rock, quartz grains undergo the $\beta-\alpha$ phase transition. These grains are twinned according to different twin-laws. The twinning (other factors are not considered) results in a rise of the piezoelectric properties of $\alpha$-quartz texturized samples.

If crystals are twinned according to the Leidolt law (Shubnikov, 1975), the polycrystal becomes piezoelectrically active. As a result, the peizoelectric longitudinal and transversal effects must be observed. If the twinning takes part according to the Dauphine law, texturized rocks may become piezoelectrically active, if shear stresses are applied. Finally, polycrystals with grains which are twinned according to the Brazil law do not 

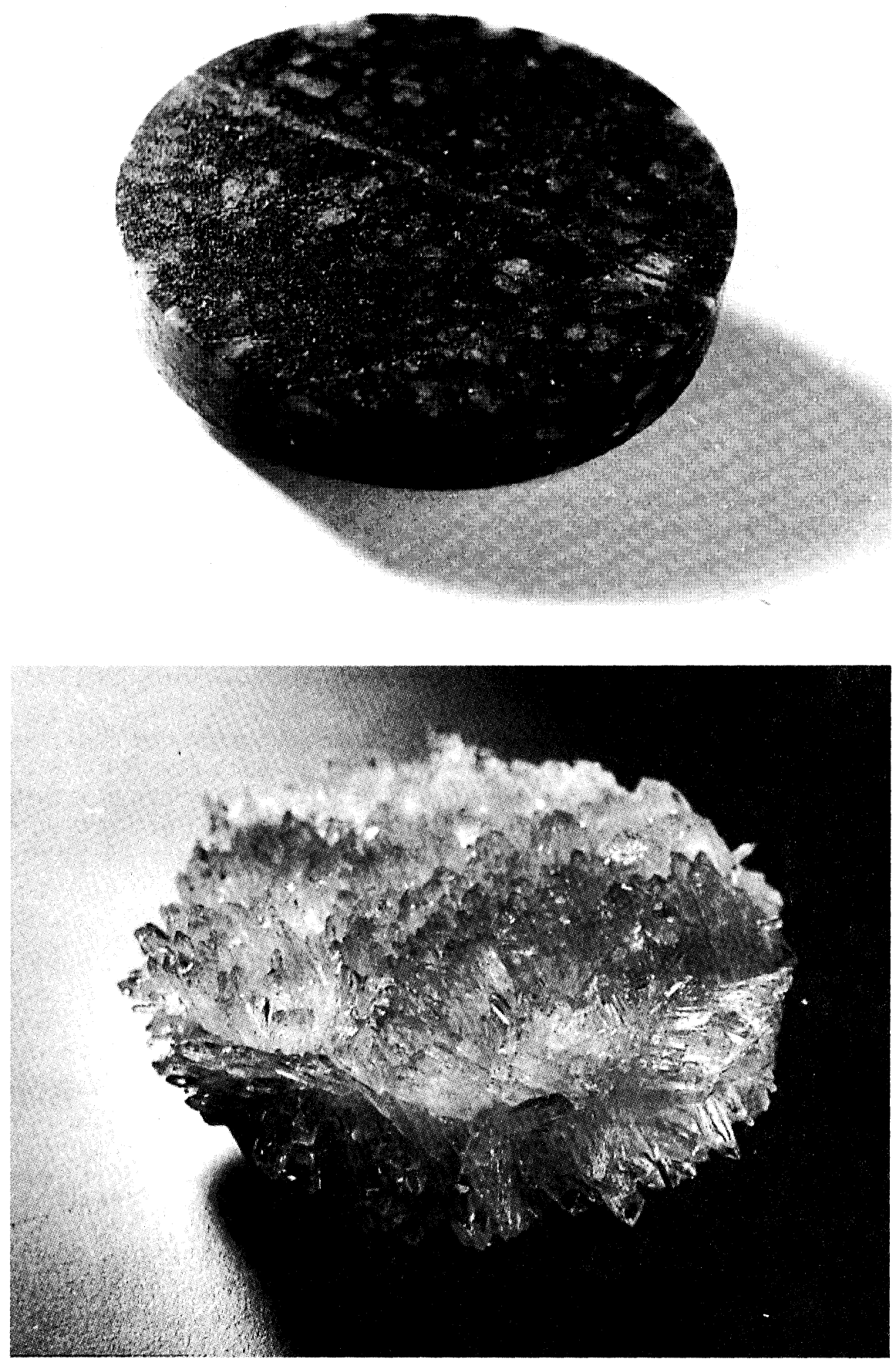

Figure 6 Photographs of a sample (a) before the experiment; (b) after the experiment. 
show any piezoelectric properties. However, the two crystals in a twin have different sizes if they grow according to the Brazil law under nonhydrostatic stresses. In this case, the differences in size will only cause partial compensation of the electric charges of the twinned crystals and the texturized rock will be piezoelectrically active. In twinned quartz polycrystals an increase in the polar preferred orientations of the same sign occurs following recrystallyzation under the action of nonhydrostatic stresses. Finally, it can be concluded that the piezoelectric properties of texturized quartz rocks can be the result of the crystallyzation or of the $\beta-\alpha$ phase transition. Moreover, in the $\beta-\alpha$ phase transition the effect of internal mechanical fields plays an important role.

\section{Piezoelectricity of rocks with sedimentation textures}

Now, the question arises whether piezoelectric properties can be also observed in sedimentary rocks. This hypotheses can be experimentally verified. The following experiment was carried out to obtain sedimentation textures.

A synthetic quartz monocrystal was turned into powder, and a fraction of this powder having grains with the size of $50 \mu \mathrm{m}$ down to $20 \mu \mathrm{m}$ was separated by sifting. Then, the quartz powder was sedimented in $250 \mathrm{~mm}$ high alcohol-filled glass retort a with pyrophilite envelope at the bottom. The lower hole of the retort and the envelope were sealed using a special plastic composite. In the liquid column, an electric field was generated by two flat electrodes $300 \mathrm{~mm}$ height with a diameter $60 \mu \mathrm{m}$ connected to a static polarizer. The voltage across the electrodes varied from 1000 to $5000 \mathrm{~V}$. The quartz particles were previously heated to $400-500^{\circ} \mathrm{C}$ and then sedimented in the glass-retort at $20^{\circ} \mathrm{C}$. At fast cooling, elastic stresses appeared in quartz grains. Due to the piezoelectric effect, electric polarization arises in every grain, and the direction of the electric moment arranges with the direction of electric axes in quartz. During sedimentation the grains acquire a dipole moment and orient according to the polarization of the external electric field.

The preferred orientations of the electric axes in thus formed polycrystal quartz aggregates were determined by inverse pole figure X-rays measurements. Figure 7 shows the inverse pole figure for the direction, which coincides with the axis of the cylindrical sample. The increase in the pole density towards the $\{11 \overline{2} 0\}$ direction can be interpreted as an alignment of electric axes of the quartz grains in the sample.

From the experiments it can be concluded that the piezoelectric activity of sediments indicates some conditions which should be fulfilled in the genesis of rocks, e.g., the presence of a permanent electric field within the ancient basin for a long time and of a mechanism which causes the polarization of piezoelectric active particles during sedimentation.

\section{CONCLUSIONS}

Finally, it should be emphasized that studies of the texture-induced piezoelectric effect of rocks give us the key to the history of rocks.

We suggest three probable mechanisms of the formation of piezoelectric active rocks with textures:

- during crystallization of quartz from a hydrothermal solution;

- twinning, when deformed vein quartz experiences $\beta-\alpha$ phase transition.

- during sedimentation. 


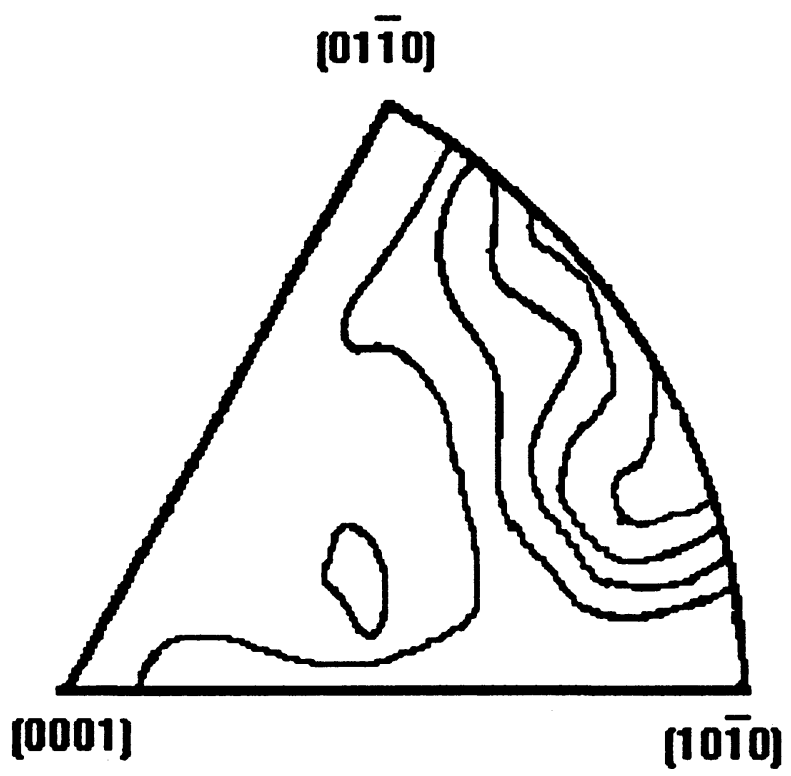

Figure 7 Inverse pole figure of the sample cylinder axis obtained from the DRON-2 X-ray diffractometer.

\section{Acknowledgements}

The authors express their deep appreciation to V. E. Hadgi and L. I. Zinober for support to the experiments on synthetic quartz growth made at the Russian Research Institute for the Synthesis of Minerals (RNIISIMS).

\section{References}

Bishop, J. R. (1981). Piezoelectric effects in quartz-rich rocks. Tectonophysics, 77, 297-321.

Ivankina, T. I., Nikitin, A. N. and Ullemeyer, K. (in press). The reconstruction of the geodynamic state of rocks in the lithosphere by means of texture analysis. Journal of earthquake prediction research.

Ivankina, T. I., Nikitin, A. N., Voitus, W. and Walther, K. (1991). Texture analysis and investigation of piezoelectric properties of natural quartz. Textures and Microstructures, 14-18, 421-429.

Nikitin, A. N., Volarovich, M. P., Parkhomenko, E. I. and Golikov, M. I. (1981). Measurement of the angular dependences of the piezoeffect in samples possessing piezoelectric activity. Izvestiya AN SSSR, Fizika Zemli, 2, 36-44.

Nikitin, A. N. and Parkhomenko, E. I. (1982a). Study of the effect of defects and imperfections in the orientation of the quartz structure on the manifestation of the piezoelectric effect. Physics of the Solid Earth, published by AGU and GSA, 18, 1, 75-82.

Nikitin, A. N. and Parkhomenko, E. I. (1982b). Piezoelectric effect of quartz-bearing rocks and their symmetry properties. Physics of the Solid Earth, published by AGU and GSA, 18, 2, 104-110.

Royburt, A. L. (1983). Influence of mechanical stresses on the generation of domain structures under martensite and segnetoelectric phase transformations. Izvestiya AN SSSR, Serya Fizika, 47, 3, 27-40.

Shubnikov, A. V. (1946). Piezoelectric textures. Moscow-Leningrad: Izd-vo AN USSR, 99p.

Shubnikov, A. V. (1975) Selected works on crystallography. M., Nauka, 217-223. 
Tyck, G. I., Stace, F. O. and Starkey, A. (1977). A search for the piezoelectric effect in quartz-bearing rocks. Tectonophysics, 39, 4, 175-179.

Volarovich, M. P. and Parkhomenko, E. I. (1954). The piezoeffect in rocks. Dokl. AN SSSR, 90, 2, 239-242.

Walther, K., Nikitin, A. N., Voitus, W. and Ivankina, T. I. (1990). Study of textures of quartz rocks by neutron diffraction. Izvestiya AN USSR, Fizika Zemli, 11, 78-84.

Walther, K., Nikitin, A. N., Isakov, N. N., Ullemeyer, K. and Heinitz, J. (1993). Diffraction studies of textures in geomaterials using a high-resolution neutron spectrometer. Izvestiya AN USSR, Fizika Zemli, 6, 37-44.

Walther, K., Kurtasov, S.F., Nikitin, A. N. and Torina, Ye. G. (1994). Simulation of deformation textures in high-temperature quartz. Physics of the Solid Earth, published by $A G U$ and GSA, 29, 6, 497-500.

Wenk, H.-R. (1993). Texture analysis with TOF neutrons. Time-of-flight diffraction at Pulsed Neutron Sources. TACA, 29, 95-108.

Zinober, L. I., Hadgi, V. E., Samoylovich, M. I. and Balizky, V. S. (1972). Growth conditions and the real structure of quartz. Proceedings of the IV Joint workshop on growing crystals, part II, 186-205. 